INNOVATIONS IN PRIMARY CARE

\title{
Population Mapping for Quality Improvement in a Neigh- borhood Health Center
}

\author{
Autumn M. Kieber-Emmons, MD, MPH ${ }^{1-3}$ \\ Michael Topmiller, $\mathrm{PbD}^{3}$ \\ Mark A. Carrozza, $M A^{3}$
}

Ann Fam Med 2020;18:374. https://doi.org/10.1370/afm.2531.

\section{THE INNOVATION}

Our health center has been using a population-health mapping tool (HealthLandscape's Population Health Profiler') to better understand health disparities for our service area, to target resources for care in our community, and to conduct comparative research to improve health outcomes for our patients.

\section{WHO AND WHERE}

Neighborhood Health Centers of the Lehigh Valley Federally Qualified Health Center is located in Allentown, Pennsylvania, a diverse city (over $42 \%$ Hispanic) located 2 hours outside of New York City. The center has served over 10,000 vulnerable and at-risk patients in Allentown since 2016, many of whom have sociodemographic barriers related to insurance, language, finances, transportation, education, and housing.

HealthLandscape's Population Health Profiler is an innovative geospatial mapping tool developed by the American Academy of Family Physicians (AAFP). The tool allows users to upload patient data aggregated to the zip code level to create service area maps, and to explore a multitude of "community vital signs" including basic demographic and economic measures, Social Deprivation Index (SDI) ${ }^{2}$ and inequality, chronic conditions, and access to care. The maps and detailed reports can be used to display the geographic reach of a health center, highlight neighborhoods with high-risk populations, map chronic disease hot spots, and identify access barriers to reach providers.

\section{HOW}

We have used the Population Health Profiler for projects focused on cancer disparities, diabetes hot spots, and prenatal

Conflicts of interest: autbors report none.

\section{CORRESPONDING AUTHOR}

Autumn M. Kieber-Emmons, MD, MPH

Lehigh Valley Health Network

Department of Family Medicine

PO Box 1806

Allentown, PA 18105

Autumn.kieber-emmons@lvhn.org care provision within our community. We began using this mapping tool with a large funded study on cancer mortality disparities. We uploaded health center data into the Profiler to create maps of all of the cancer patients' zip codes by neighborhood and survival status, and then used the Profiler's community vital sign tools to clarify areas of highest need based on vulnerable demographic and economic markers. The center used this information in quality improvement development of a new cancer survivorship clinic held every month within 2 of 3 sites for our center. Additionally, the center data was compared across other states from the funded study to better understand regional differences in sociodemographic and medical variables of interest related to cervical cancer deaths in Hispanic women.

We then became interested in how the Population Health Profiler could be used for multiple quality improvement projects, with our quality registered nurse (RN) and doctor of medicine (MD) creating maps from uploaded zip code data from the medical records. We have since expanded use of the mapping tool to begin mapping diabetics by zip code to identify hot spots of diabetes for community outreach to those areas. Additionally, our maternal health quality committee (1 midwife, 2 nurses, 1 social worker, 4 physicians, and $1 \mathrm{MPH}$ data analyst) are using the tool to better understand changes in the service area of our prenatal patients over the last 5 years. These data are being used to design quality improvement goals for our prenatal care program, including outreach within communities to increase patient volume of prenatal patients, a home visiting postpartum nurse program, using maps to help identify priority prenatal community resource agency partners within our highest volume zip codes, and using service area maps to unroll Centering ${ }^{\mathrm{TM}}$ prenatal group care in a second site.

\section{LEARNING}

HealthLandscape's Population Health Profiler is an easy-to-use clinical and population health tool that can assist any sized clinics with population health management. Through the creation of maps and reports that showcase neighborhoods with the highest vulnerability, health centers or other clinics can focus resources and services to design community-specific quality improvement projects to address high-need areas for your patient population. The Profiler can assist with quality improvement for a variety of chronic diseases, as well as provide a platform for comparison of sociodemographic variables across regions.

For supplemental information, including author affiliations, key words, and references, see https://www.AnnFamMed.orgl content/18/4/374/suppI/DC1. 